\title{
Corneal edema recovery after phacoemulsification in type 2 diabetic versus non-diabetic patients
}

\section{ABSTRACT}

Background. Ultrasound energy during phacoemulsification can cause mechanical trauma to corneal endothelium which leads to prolonged postoperative recovery. Several studies have reported conflicting results of corneal changes after phacoemuslification in diabetic versus non-diabetic patients. We aimed to assess corneal edema recovery and compare best corrected visual acuity (BCVA) after phacoemulsification in patients with diabetes mellitus type 2 versus patients without type 2 diabetes.

Methods. The study included type 2 diabetic and nondiabetic patients who underwent phacoemulsification between November 2018 and May 2019. Assessments of corneal edema recovery were done preoperative and on postoperative day 1 , after 1 week and after two months. BCVA were taken preoperative and postoperative after 1 week and 2 months after the surgery. Results. There was no significant difference between groups in corneal status on check-up examinations. Corneal edema recovery was equal between the patients with and without diabetes mellitus type 2 . No significant difference in mean BCVA was observed between the studied groups after the operation.

Conclusion. Considering the use of modern technology in cataract surgery, there was no difference in

Address for correspondence:

Martina Liovic Milec

Eye Department, General Hospital dr. Josip Bencevic,

Josipa Kozarca 4, 35208 Ruscica, Croatia

e-mail: martinaliovic@yahoo.com

Clinical Diabetology 2021, 10, 1: 144-148

DOI: $10.5603 /$ DK.a2021.0003

Received: 07.08.2020

Accepted: 27.12.2020 postoperative results between non-diabetic and type 2 diabetic patients. (Clin Diabetol 2021; 10, 1: 144-148)

Key words: cataract, phacoemulsification, corneal edema, diabetes mellitus, visual acuity

\section{Introduction}

Diabetes mellitus (DM) is a metabolic disease with long-term consequences for many organs and cataract is one of the earliest DM complications. There is an increase in the world diabetes prevalence from 9.3\% in 2019 to $10.9 \%$ in 2045 due to population growth, ageing, urbanization, sedentary lifestyles and adverse dietary changes [1]. Since it is estimated that $20 \%$ of all cataract surgeries are done on diabetic patients, there is a growing number of studies that have reported various results of phacoemulsification effect on corneal recovery in diabetic and non-diabetic patients [2, 3]. Ultrasound energy during phacoemulsification can cause mechanical trauma and induce corneal endothelial cell loss which can lead to slower postoperative corneal recovery. In this study we aimed to evaluate corneal edema recovery and compare best corrected visual acuity (BCVA) after phacoemulsification in type 2 diabetic and non-diabetic patients.

\section{Methods}

This prospective cohort study was conducted to assess corneal edema recovery and compare BCVA after phacoemulsification in type 2 diabetic versus non-diabetic patients.

The study included 267 patients (209 non-diabetic and 58 type 2 diabetic eyes) selected to undergo phaco- 
emulsification surgery between November 2018 and May 2019 at the Department of Ophthalmology, GH dr. Josip Bencevic, Slavonski Brod, Croatia.

The patients underwent preoperative ophthalmological examination, including BCVA using Snellen chart and then analyse the data after converting it to logMAR (log of the Minimum Angle of Resolution), Goldmann applanation tonometry, slit lamp examination, dilated indirect fundus examination, preoperative central corneal thickens (CCT) measurements by IOL-Master V.5 (Carl Zeiss Meditec AG). Medical history (age, sex, prior eye disease, previous diseases and medication) was documented. Exclusion criteria were the patients with a history of previous ocular surgery or inflammation and Fuchs' endothelial dystrophy.

Cataract density was graded according to the Lens Opacities Classification System III (LOCS III) using NO (1-6) as a grading scale [4]. The examined eyes were subdivided into mild (NO1, NO2), moderate (NO3, NO4) and hard (NO5, NO6) cataracts.

All procedures were performed by one experienced surgeon (S.S.). Phacoemulsification was performed by WhiteStar Signature ${ }^{\circledR}$ PRO system using phaco-chop technique with Ellips FX handpiece (AMO, Inc.). The intraocular lens was placed within the capsular bag in all cases.

Postoperative treatment included the use of dexamethasonum/neomycinum/polymyxinum B (Maxitrol) drops four times per day for the first week and three times per day until week 4 . All type 2 diabetic patients also used bromfenac drops two times per day starting three days before the surgery and continuing for one month.

Patients were followed on postoperative day 1 , after 1 week and after two months. Clinical measurements included postoperative cornea examination using 3 grading scales: clear cornea - transparent cornea without Descemet' $s$ folds, and other two when corneal edema is present according to the Oxford Cataract Treatment and Evaluation Team (OCTET): focal corneal edema - transient corneal oedema with Descemet membrane folds of $<10$, diffusive corneal edema transient corneal oedema with Descemet membrane folds of $>10$ [5]. The state of the cornea was noted in each follow-up. Postoperative BCVA was taken on postoperative day 7 and after two months.

Out of 267 patients enrolled, 266 patients came to check-up on postoperative day 1 (208 non-diabetic and 58 type 2 diabetic eyes), 265 patients on postoperative day 7 (208 non-diabetic and 57 type 2 diabetic eyes) and after two months 205 patients (159 non-diabetic and 49 type 2 diabetic eyes) came to our hospital to check-up. Other patients went to other hospitals on follow up. Among the 58 type 2 diabetic patients,
Table 1. Distribution of cataracts according to LOCS III grading in type 2 diabetic patients (study group) and non-diabetic patients (control group)

\begin{tabular}{lcccc}
\hline & \multicolumn{2}{c}{$\begin{array}{c}\text { Study group } \\
(\mathbf{n}=\mathbf{5 8})\end{array}$} & \multicolumn{2}{c}{$\begin{array}{c}\text { Control group } \\
(\mathbf{n}=209)\end{array}$} \\
\cline { 2 - 5 } & $\mathbf{n}$ & $\%$ & $\mathbf{n}$ & $\%$ \\
\hline LOCS III & & & & \\
Mild cataract & 4 & 6.9 & 25 & 12.0 \\
Moderate cataract & 42 & 72.4 & 138 & 66.0 \\
Hard cataract & 12 & 20.7 & 46 & 22.0
\end{tabular}

$\mathrm{N}$ - number

43 had no diabetic retinopathy (DR) and 15 had mild nonproliferative diabetic retinopathy (NPDR).

The study was performed in accordance with the 1964 Declaration of Helsinki. Informed consent was obtained from all patients.

\section{Statistical analysis}

Statistical analysis was performed using SPSS software (version 24, SPSS INC, IBM Coropartion, Chicago, USA): significance was tested by using Wilcoxon $\mathrm{W}$, chi-square test and Friedman test. P-values $<0.05$ were considered statistically significant.

\section{Results}

This prospective cohort study was conducted to assess corneal edema recovery and compare BCVA after phacoemulsification in type 2 diabetic patients versus non-diabetic patients.

The average age of the study population was 73. Most patients had moderate cataract in type 2 diabetic patients $(72.4 \%)$, as well as in non-diabetic patients $(66.0 \%)$. There was no statistically significant difference in cataract density according to the LOCS III grading between both groups $\left(\chi^{2}, p=0.501\right)$ (Table 1 ). Median preoperative CCT was 553 . There was no significant difference in preoperative logMAR BCVA between the patients with and without diabetes mellitus type 2 (Table 2).

Visual acuity statistically varied during three interval periods. There was significant improvement in logMAR BCVA (Friedman test, $p<0.001$ ) with no significant difference in postoperative BCVA between groups, as seen in Table 2. We compared BCVA in patients with hard cataract since this group was in the high risk and there was no statistically significant difference in BCVA in non-diabetic versus type 2 diabetic patients (Table 3).

Most of the patients had clear cornea in both groups on days 1 and 7 after the operation. There 
Table 2. Preoperative (BCVA1), 1 week postoperative (BCVA2) and 2 months postoperative (BCVA3) logMAR best corrected visual acuity in all type 2 diabetic patients (study group) and non-diabetic patients (control group)

\begin{tabular}{|c|c|c|c|c|c|c|c|}
\hline & \multicolumn{3}{|c|}{$\begin{array}{l}\text { Study group } \\
\qquad(n=58)\end{array}$} & \multicolumn{3}{|c|}{$\begin{array}{l}\text { Control group } \\
\quad(n=208)\end{array}$} & \multirow[t]{2}{*}{ P value* } \\
\hline & $n$ & $M$ & SD & $n$ & $M$ & SD & \\
\hline BCVA1 & 58 & 0.30 & 0.62 & 208 & 0.30 & 0.62 & 0.865 \\
\hline BCVA2 & 57 & 0.05 & 0.64 & 208 & 0.05 & 0.68 & 0.789 \\
\hline BCVA3 & 46 & 0.00 & 0.70 & 159 & 0.00 & 0.80 & 0.362 \\
\hline
\end{tabular}

*Wilcoxon $\mathrm{W}$ test; $\mathrm{n}$ - number; $\mathrm{M}$ - median; SD — standard deviation

Table 3. Preoperative (BCVA1), 1 week postoperative (BCVA2) and 2 months postoperative (BCVA3) logMAR best corrected visual acuity in non-diabetic (control group) and type 2 diabetic patients (study group) with hard cataract

\begin{tabular}{lccccccc}
\hline & \multicolumn{3}{c}{$\begin{array}{c}\text { Study group } \\
(\mathbf{n}=12)\end{array}$} & \multicolumn{3}{c}{$\begin{array}{c}\text { Control group } \\
(\mathbf{n}=46)\end{array}$} & P value* \\
\cline { 2 - 6 } & $\mathbf{n}$ & $\mathbf{M}$ & SD & $\mathbf{n}$ & $\mathbf{M}$ & SD & \\
\hline BCVA1 & 12 & 1.40 & 0.58 & 46 & 1.30 & 0.64 & 0.923 \\
BCVA2 & 11 & 0.00 & 0.96 & 46 & 0.05 & 0.72 & 0.342 \\
BCVA3 & 9 & 0.00 & 1.40 & 36 & 0.00 & 0.96 & 0.845 \\
\hline
\end{tabular}

*Wilcoxon $\mathrm{W}$ test; $\mathrm{n}$ - number; $\mathrm{M}$ - median; SD — standard deviation

was no significant difference between groups in the status of the cornea on check-up examinations, as seen in Table 4. There were no more cases of diffusive corneal edema in both groups on seventh postoperative day. In the study, all the examined patients (205) had clear cornea two months after the operation. We also compared corneal edema recovery in patients with hard cataract and there was no significant difference between groups (Table 5).

\section{Disscusion}

The most superficial and the most transparent organ affected by DM is the cornea, which is the topic of our research [6].

Our general hospital serves a population of near 150,000 inhabitants and in our study there were 267 patients, at the average age of 74 included. There were $58(21.7 \%)$ type 2 diabetic patients which is consistent with the data from literature estimating that the prevalence of diabetes among elderly population $\geq 65$ years old is between $22 \%$ and 33\% [7]. The global prevalence for DR is $27 \%$, out of which $25.2 \%$ is NPDR, $1.4 \%$ proliferative diabetic retinopathy (PDR) and $4.6 \%$ diabetic macular edema (DME) [8]. This prevalence for DR corroborates analogous results from our study population, there were $21.7 \%$ type 2 diabetic patients, out of which $25.9 \%$ had NPDR.

There was no difference in the postoperative BCVA after 2 months between non-diabetic and type 2 diabetic patients (median logMAR BCVA was 0.00 in both groups) in our study. Thus, type 2 diabetic patients without DR and those with mild NPDR have a chance for equal visual acuity as non-diabetic patients, even in patients with hard cataract. Similar conclusion was reached by the authors who emphasize that with a careful preoperative examination and DR pretreatment, as well as with minimally invasive surgical techniques in cataract operation diabetic patients recover excellently $[9,10]$

Second aim of this study was to observe the postoperative recovery of corneal edema and there was no difference between non-diabetic and type 2 diabetic patients. The prevalence and corneal edema was approximately the same in both studied groups on all check-up examinations. On the first postoperative day, focal corneal edema was present in one-quarter of patients in both groups, while diffusive corneal edema

Table 4. Corneal edema recovery between type 2 diabetic patients (study group) and non-diabetic (control group)

\begin{tabular}{|c|c|c|c|c|c|}
\hline & \multicolumn{2}{|c|}{ Study group $(n=58)$} & \multicolumn{2}{|c|}{ Control group $(n=208)$} & \multirow[t]{2}{*}{ P value ${ }^{*}$} \\
\hline & $n$ & $\%$ & $\mathrm{n}$ & $\%$ & \\
\hline \multicolumn{6}{|l|}{1 day postoperative } \\
\hline Clear cornea & 39 & 67.2 & 134 & 64.4 & \\
\hline Focal corneal edema & 14 & 24.1 & 54 & 26.0 & 0.922 \\
\hline Diffusive corneal edema & 5 & 8.6 & 20 & 9.6 & \\
\hline \multicolumn{6}{|l|}{7 days postoperative } \\
\hline Clear cornea & 54 & 94.7 & 201 & 96.6 & \\
\hline Focal corneal edema & 3 & 5.3 & 7 & 3.4 & 0.505 \\
\hline \multicolumn{6}{|l|}{2 months postoperative } \\
\hline Clear cornea & 46 & $100.0 \%$ & 159 & 100.0 & \\
\hline
\end{tabular}

${ }^{*} \chi^{2} ; \mathrm{n}-$ number 
Table 5. Corneal edema recovery between type 2 diabetic patients (study group) and non-diabetic (control group) with hard cataract

\begin{tabular}{|c|c|c|c|c|c|}
\hline & \multicolumn{2}{|c|}{ Study group $(n=12)$} & \multicolumn{2}{|c|}{ Control group $(n=46)$} & \multirow[t]{2}{*}{ P value } \\
\hline & $\mathrm{n}$ & $\%$ & $n$ & $\%$ & \\
\hline \multicolumn{6}{|l|}{1 day postoperative } \\
\hline Clear cornea & 7 & 58.3 & 21 & 45.7 & \\
\hline Focal corneal edema & 3 & 25.0 & 16 & 34.8 & 0.498 \\
\hline Diffusive corneal edema & 2 & 16.7 & 9 & 19.6 & \\
\hline \multicolumn{6}{|l|}{7 days postoperative } \\
\hline Clear cornea & 10 & 90.9 & 44 & 95.7 & \\
\hline Focal corneal edema & 1 & 9.1 & 2 & 4.3 & 0.530 \\
\hline \multicolumn{6}{|l|}{2 months postoperative } \\
\hline Clear cornea & 9 & 100.0 & 36 & 100.0 & \\
\hline
\end{tabular}

*Wilcoxon W test; $\mathrm{n}$ - number

was present in a smaller number of non-diabetic $(9.6 \%)$ and type 2 diabetic patients (8.6\%). On the check-up examination after 7 days, there was a significant improvement in the clinical picture and corneal edema recovery, only a small number of patients had focal corneal edema $(3.4 \%$ of the non-diabetic patients, $5.3 \%$ of the type 2 diabetic patients). Consumed ultrasound energy during phacoemulsification can make endothelial injury which can lead to postoperative transient corneal edema after operation. The amount of phacoemulsification energy increases due to increasing grades of cataract density, so patients with hard cataract are in higher risk to get transient postoperative edema and to have slower corneal edema recovery. The results of this study showed that patients with hard cataract had more postoperative corneal edema in nondiabetic and type 2 diabetic patients, as seen in Tables 4 and 5 . On the first postoperative day, the number of patients with diffusive corneal edema was doubled when we compared patients with hard cataract with all the studied patients, non-diabetic (16.7\% and 9.6\%) and type 2 diabetic patients (19.6 and 8.6\%). But it is important for our study that patients who had hard cataract with or without diabetes mellitus type 2 had similar corneal edema recovery and good postoperative visual acuity (median logMAR BCVA after 2 months was 0.00 for both groups) as seen in all the studied patients. This can be due to reason that we used phaco-chop technique to have minimally invasive cataract operation. It has been shown that less ultrasound energy is needed when using phaco-chop in comparison with other techniques [11]. In conclusion, corneal edema was present almost equally in non-diabetic and type 2 diabetic patients and it also recovered in the same time period in both studied groups.
Similar to our study, Kausar et al. did not find the link between corneal edema and DM either, with the remark that their study included a small number of patients with DM [11]. Al-Sharkawey et al. reached the conclusion that endothelial cell loss (ECL) is $8 \%$ in all patients, regardless of DM [12]. Also, Budiman reported that there were no differences in endothelial cell density between diabetic and non-diabetic group and he concluded that $\mathrm{HbA}_{1 \mathrm{c}}$ level in diabetic patients did not effect on corneal endothelium [13]. Unlike our study, some studies concluded that corneal edema is more common in diabetic patients than in non-diabetic patients $[14,15]$ and also many studies have reported higher ECL in patients with DM, a factor that affects slower recovery of the cornea in the initial postoperative period [2, 16-19].

However, Hugod et al. concluded that there is a higher ECL in patients with DM, but the functional corneal status regarding CCT and visual acuity is approximately the same as in non-diabetic patients [10]. Consequently, except for endothelial cells quantity, the quality of endothelial cells is also an important factor for corneal transparency.

The limitations of this study were a short followup period, lack of specular microscope chart due to lack of resources, lower grades of retinopathy and fair glycaemic control.

In conclusion, cataract surgery is followed by approximately equal postoperative results in patients with or without diabetes mellitus type 2 , even in patients with hard cataract. With the use of modern technology and with minimally invasive surgical techniques in cataract surgery, a good prognosis can be expected in the postoperative recovery in type 2 diabetic population without or with mild diabetic retinopathy. 


\section{Conflict of interest}

Authors have no conflicts of interest to disclose.

\section{REFERENCES}

1. Saeedi P, Salpea P, Karuranga S, et al. Global and regional diabetes prevalence estimates for 2019 and projections for 2030 and 2045: Results from the International Diabetes Federation Diabetes Atlas, 9 edition. Diabetes Res Clin Pract. 2019; 157: 107843, doi: 10.1016/j.diabres.2019.107843, indexed in Pubmed: 31518657.

2. Javadi MA, Zarei-Ghanavati S. Cataracts in diabetic patients: a review article. J Ophthalmic Vis Res. 2008; 3(1): 52-65, indexed in Pubmed: 23479523.

3. Tang $Y$, Chen $X$, Zhang $X$, et al. Clinical evaluation of corneal changes after phacoemulsification in diabetic and non-diabetic cataract patients, a systematic review and meta-analysis. Sci Rep. 2017; 7(1): 14128, doi: 10.1038/s41598-017-14656-7, indexed in Pubmed: 29074989.

4. Chylack L. The Lens Opacities Classification System III. Archives of Ophthalmology. 1993; 111(6): 831, doi: 10.1001/ archopht.1993.01090060119035.

5. Use of a grading system in the evaluation of complications in a randomised controlled trial on cataract surgery. Oxford Cataract Treatment and Evaluation Team (OCTET). Br J Ophthalmol. 1986; 70(6): 411-414, doi: 10.1136/bjo.70.6.411, indexed in Pubmed: 2872914.

6. Shih KCo, Lam KSL, Tong L. A systematic review on the impact of diabetes mellitus on the ocular surface. Nutr Diabetes. 2017; 7(3): e251, doi: 10.1038/nutd.2017.4, indexed in Pubmed: 28319106.

7. Kirkman MS, Briscoe VJ, Clark N, et al. Diabetes in older adults. Diabetes Care. 2012; 35(12): 2650-2664, doi: 10.2337/dc121801, indexed in Pubmed: 23100048.

8. Thomas RL, Halim S, Gurudas S, et al. IDF Diabetes Atlas: A review of studies utilising retinal photography on the global prevalence of diabetes related retinopathy between 2015 and 2018. Diabetes Res Clin Pract. 2019; 157: 107840, doi: 10.1016/j. diabres.2019.107840, indexed in Pubmed: 31733978.

9. Kelkar A, Kelkar J, Mehta H, et al. Cataract surgery in diabetes mellitus: A systematic review. Indian J Ophthalmol. 2018; 66(10): 1401-1410, doi: 10.4103/ijo.IJO_1158_17, indexed in Pubmed: 30249823.
10. Hugod M, Storr-Paulsen A, Norregaard JC, et al. Corneal endothelial cell changes associated with cataract surgery in patients with type 2 diabetes mellitus. Cornea. 2011; 30(7): 749-753, doi: 10.1097/ICO.0b013e31820142d9, indexed in Pubmed: 21317781.

11. Kausar A, Farooq S, Akhter W, et al. Transient corneal edema after phacoemulsification. J Coll Physicians Surg Pak. 2015; 25(7): 505509, doi: 07.2015/JCPSP.505509, indexed in Pubmed: 26208554.

12. Al-Sharkawy H. Corneal endothelial changes in type 2 diabetes mellitus before and after cataract surgery. Journal of the Egyptian Ophthalmological Society. 2015; 108(2): 79, doi: 10.4103/20900686.161400.

13. Budiman B. Comparison of endothelial cell density, morphological changes and central corneal thickness after phacoemulsification between diabetic and non-diabetic patients. The Open Ophthalmology Journal. 2020; 14(1): 15-20, doi: $10.2174 / 1874364102014010015$.

14. Tsaousis KT, Panagiotou DZ, Kostopoulou E, et al. Corneal oedema after phacoemulsification in the early postoperative period: A qualitative comparative case-control study between diabetics and non-diabetics. Ann Med Surg (Lond). 2016; 5: 67-71, doi: 10.1016/j.amsu.2015.12.047, indexed in Pubmed: 26865977.

15. Shakya K, Pokharel S, Karki KJD, et al. Corneal edema after phacoemulsification surgery in patients with type II diabetes mellitus. Nepal J Ophthalmol. 2013; 5(2): 230-234, doi: 10.3126/nepjoph. v5i2.8734, indexed in Pubmed: 24172560.

16. Khalid M, Hanif MK, Islam QUI, et al. Change in corneal endothelial cell density after phacoemulsification in patients with type II diabetes mellitus. Pak J Med Sci. 2019; 35(5): 1366-1369, doi: 10.12669/pjms.35.5.596, indexed in Pubmed: 31489008.

17. He Xu, Diakonis VF, Alavi Y, et al. Endothelial cell loss in diabetic and nondiabetic eyes after cataract surgery. Cornea. 2017; 36(8): 948-951, doi: 10.1097/ICO.0000000000001245, indexed in Pubmed: 28614156.

18. Bamdad S, Bolkheir A, Sedaghat MR, et al. Changes in corneal thickness and corneal endothelial cell density after phacoemulsification cataract surgery: a double-blind randomized trial. Electron Physician. 2018; 10(4): 6616-6623, doi: 10.19082/6616, indexed in Pubmed: 29881523.

19. El-Agamy A, Alsubaie S. Corneal endothelium and central corneal thickness changes in type 2 diabetes mellitus. Clin Ophthalmol. 2017; 11: 481-486, doi: 10.2147/OPTH.S126217, indexed in Pubmed: 28280298. 